\title{
POST-ANGINAL SEPSIS (Sepsis of Oro-Naso-Pharyngeal Origin)
}

\author{
BY \\ MARK S. REUBEN, M.D. \\ (From the Prediatric Department of College of Physicians and Surgeons, \\ Columbia University, and the Pædiatric Department of the Beth Israel \\ Hospital, New York.)
}

The belief that the so-called common sore throat is of no serious consequence is fallacious: serious complications and death follow it sufficiently often to warrant a study of the complications of simple angina and their mode of evolution. It is true that in a great majority of cases, there is a restitutio ad integrum in a short time and that the patient is able to follow his usual mode of life in a few days ; but it is equally true that in a certain number, after a period of from a few days up to from seven to eleven weeks after the angina has subsided, a group of symptoms, local and systemic (sepsis), develops which not only threatens life but may prove fatal. The evidence is cumulative and convincing. In many of these cases of post-anginal sepsis, either thrombosis (obturating or mural) or phlebitis of the internal jugular vein or its anastomosing branches was found at the operating table or on autopsy. In a number of instances of so-called idiopathic or cryptogenic sepsis, pathologists have demonstrated thrombosis of an internal jugular vein; it is deemed advisable now to explore the internal jugulars in cases of sepsis of unknown origin especially those following angina or any infection of the oro-naso-pharynx. This is considered good surgery in the field of the neck, just as it is considered advisable to explore a case of an acute surgical abdomen of unknown origin. This procedure is not only of theoretical value, for it has been carried out many times, and in cases in which the jugular and its anastomoses were ligated and excised early, the patients made rapid and complete recoveries. In many cases of sepsis following oro-naso-pharyngeal infection, where there is only phlebitis of the vein, the patients recover when the primary focus of infection is completely removed, even in the presence of metastases, without any operative procedures on the veins of the neck.

The subject deserves much greater attention than it has received. Apart from a few case reports there has not appeared a single comprehensive article on this subject in English. Most of the work has been accomplished in Germany, but the first who clearly and distinctly called attention to the correlation of primary angina and secondary sepsis associated with thrombosis of the internal jugular veins was Dr. Mosher of Boston. 


\section{Historical.}

Quinsy and phlegmonous retro-pharyngitis were known to the ancients. Hippocrates not only referred to it but emphasized the presence of opisthotonos as one of its symptoms. He suggested that such abscesses should be opened with the finger if they were soft, and if not, an iron should be attached to the finger and the abscess opened with it.

Morrell Mackenzie stated that the first definite mention of abscess in the retro-pharyngeal region was made by Galen in the second century. He described a case with spontaneous rupture and stated that he had seen cases which resulted fatally.

Morgagni in the 18th century, gave a careful description: he cited a fatal case which ruptured into the trachea. In 1818, Abercrombie gave an accurate description of three cases in children. Henoch in 1851 gave credit to Fleming for first accurate description ; the latter devised an instrument for opening these abscesses. In 1867 Gillette demonstrated the lymph nodes and vessels of the retro-pharyngeal space. In 1858 Bokai published a comprehensive manuscript on the subject. In 1859 (hassaignac ligated the carotid artery in a case of hæmorrhage following incision of retro-pharyngeal abscess. In 1897 ('troosdinsky reported a case of extensive hæmorrhages into the skin, with staphylococei in the blood and suppurations of various organs. traceable to an old retro-pharyngeal abscess. Tischulkin in 1897 , reported fatal cases of bacterial sepsis as a result of tonsillar infections.

In 1914 Proskauer could collect only four cases of sepsis following peritonsillar abscess. (ases were also reported by Tollens (1902), H. J. Davis (1911), John W. Long (1912), H. Martin (1913) and Goodman (1917).

Mosher of Boston was the first to correlate the internal jugular vein thrombosis with the existence of the retro-pharyngeal abscess and wrote as follows :-

- Every case of peri-tonsillar or retro-pharyngeal abscess, active or quiescent, that has chills or shows a septic temperature, probably has a thrombosis of the internal jugular vein. and should be treated accordingly. When there has been a previous throat infection which has cleared up, but the patient develops a swelling of the side of the neck with a septic temperature and chills, the same condition prevails. Never let such patients die without uncovering the internal jugular vein and determining its condition.'

Subsequently Frænkel, Reye, Lewin and Rossle, Martens, Press, Melchin, Keppler and ('lairmont Welte, Wessely, Hoandt, Uffenorde, Kelemen, Kunck, Zange, Claus, Waldapfel and Riecke have all written on the subject.

\section{Portals of entry and their correlation.}

There are many portals of entry in the naso-oro-pharynx which may give rise to foci of infection and which may terminate in jugular thrombosis and sepsis. The most important are (1) the Waledeyer ring of lymphoid tissue (palatine tonsils, pharyngeal tonsil, lingual, and lymphoid tissue in the fossa of Rosenmuller and elsewhere); (2) from the maxillæ, sinus, teeth, gums ; (3) from the middle ear, mastoid and venou; sinuses, and (4) from the salivary glands, especially the parotid. It is most important to know the anatomical relationships of these parts to one another, to understand the possible involvement of all of these portals of entry secondarily to involvement of any one of them. Thus, mastoiditis may follow retro-pharyngeal abscess, and retropharyngeal abscess may follow mastoiditis by retrograde thrombosis; suppurative parotitis frequently follows otitis media purulenta and infection in the oro-naso-pharynx. Infection of the Waldeyer ring leads to para-sinusitis and this to otitis media. Ludwig's angina, peri-tonsillar abscess, retropharyngeal abscess and para-pharyngeal abscess are nature's reaction to a local infection, depending on the localization of the infection and on the peculiarity of anatomic structure of the parts involved. On final analysis, 
it is all a matter of lymphatic drainage of different areas; depending on the area involved we may have any one or all of the conditions enumerated. They are nature's second line of defence. If the infection be located in the floor of the mouth or gums, we have the possible development of Ludwig's angina; if in the posterior naso-pharynx we may have a retro-pharyngeal abscess ; if in in the area of the tonsils, we may have peri-tonsillar abscess or abscess in the tonsil ; and the last two may spread to the para-pharyngeal space.

Besides the suppurative process which may occur in these parts we may have two other conditions, depending on the severity of the infection and the resistance of the patient. In many cases, there is only hypertrophy of regional glands without suppuration, and often these masses are needlessly incised; thus, in many cases of acute infection of the adenoids, the retro-pharyngeal glands may be very much enlarged, and may produce a clinical picture not unlike that of meningitis, and yet there may be complete resolution without suppuration if the primary focus of infection in the posterior nares be promptly and properly met by suction or washing, or by emptying pockets of pus in the folds of adenoid tissue by manipulation with the finger in the posterior naso-pharynx.

On the other hand, in a case of severe infection of these parts in an individual with diminished resistance we may have a condition simulating a phlegmon of the throat in which all parts are equally affected, and there is a brawny induration of all anatomic parts of the throat and neck. This is a very serious condition and almost always proves fatal unless relieved by prompt surgery.

\section{The evolution of sepsis.}

To understand the evolution of sepsis in these cases of infection in the oro-naso-pharynx we must have a clear understanding of what is meant by retrograde thrombo-phlebitis and sepsis.

The process of retrograde thrombo-phlebitis implies the inflammation of a vein and its occlusion by a thrombus, which progresses in a direction opposite to the blood current. Thus the small venules of the tonsil may become thrombosed and this process, may extend to the jugular vein and the venous sinuses of the head.

By sepsis is meant the invasion of the blood stream by bacteria and their lodgement in tissues (reticulo-endothelial system) where they are either destroyed with the liberation or formation of a toxin, or where they form metastases. The mere presence of bacteria in the blood does not constitute sepsis; it simply implies the presence of a bacteriæmia. This occurs at the onset of many infectious diseases.

Bacteria do not long survive nor increase in number in the blood except in pre-agonal conditions. The type of fever curve seen in sepsis depends on the number of bacteria and the periodicity of invasion of the blood stream; a chill usually occurs one to two hours after an invasion of many bacteria in the blood and is the result of the reaction between them and the tissues with the formation and liberation of toxin in the blood. The bacteria do not live much longer than 15 to 30 minutes in the blood after invasion.

For the occurrence of sepsis the bacteria must be in the blood vessels. In lymphangitis the blood is usually sterile. It is only when lymphoid tissues break down and the microorganisms gain entrance into the blood stream that sepsis results : as long as the lymphatic 
and iymph glands stem the tide of infection and protect the blood stream, the infection is local and there is no sepsis.

The bacteria are destroyed in the parenchymatous organs through phagocytosis not by the leucocytes, but by the fixed tissue cells of the reticulo-endothelial system (the histiocytes). It is the cellular and not the humoral forces of resistance which are called upon mainly to destroy micro-organisms. For every 1,000 colonies that are found in $1 \mathrm{c.cm}$. of venous blood, there are probably 500 millions of bacteria in circulation. In a healthy individual they disappear within 15 minutes from the blood. Therefore it is not their appearance in the blood but their deposition in the tissues and in the peritoneum that is dangerous. Human blood in vitro also destroys bacteria; one hundred bacteria in $5 \mathrm{c.cm}$. of blood are either destroyed or become inactive. Every infectious fever is brought about by invasion of the blood or lymph by micro-organisms, and the height of the temperature depends on their number and activity. A sudden inflow of bacteria into the blood stream is followed within one to two hours by a chill and high fever; the duration and variability of temperature depend on the duration of the bacterio-lymph and on the bacteriæmia. A drop in temperature in these cases indicates cessation of inflow or conquest of bacteria. The fever curve is a true picture of the number, of the virulence and the duration of blood invasion by bacteria. A local infection, however extensive, does not give rise to fever when few or no bacteria make their way into the blood stream, even though virulent bacteria are present in the focus of infection. The mere presence of bacteria in the lymph or blood does not produce fever; this occurs only when there is liberated an endotoxin as a result of their destruction in the tissues. In every case of sepsis a focus of infection is present (though not always demonstrable). The most common foci of infection are phlebitis, thrombo-phlebitis or lymphangitis. In lymphangitis there are few or no bacteria in the blood. A suppurating lymphatic gland is only a local infection as long as it does not break down into a vein ; when this occurs it becomes a septic focus. Contrariwise a phlebitis is always a septic focus.

In many cases of post-anginal sepsis there is only found a thrombosis of the retro-tonsillar veins. Thrombosis of the jugulars is found only in a minority of the cases. In the majority of cases there is a small abscess formation behind the tonsils ; in a smaller number of cases there is thrombosis of small venous radicles. Abscess formation frequently occurs without thrombosis, but thrombosis without abscess formation practically never occurs.

In sepsis of post-anginal origin there is a considerable difference of opinion as to mode of involvement of the jugular vein where it becomes thrombosed. One group of observers believes that there is a direct extension of the thrombosis from the smallest venules of the tonsils to the jugular by its anastomosing branches; another group believes that the infections spread by lymphatics and eventually involve the veins. This discussion is of purely academic interest. Eventually there must be bacteriæmia if sepsis is to result; whether the blood stream becomes primarily involved through the smallest venules or secondarily by the breaking through of a suppurating lymph gland is of no significance for the evolution of sepsis; the bacteria must find their way into the blood stream eventually.

It is also important to differentiate between the portal of entry and the focus of infection. In many cases they are not identical. This knowledge is essential for the proper treatment. The tonsil may be the portal of entry; the focus of infection may be a peri-tonsillar abscess. In many cases the portal of entry and the focus of infection are identical. The removal of the tonsils in a certain number of cases of sepsis without accompanying peritonsillar abscess has brought about immediate relief and prompt recovery from the sepsis. 


\section{Anatomical.}

To understand the relationship of peri-tonsillar abscess to abscesses in the retro-pharyngeal and para-pharyngeal spaces, one has to be familiar with the anatomical relationship of these spaces.

A peri-tonsillar abscess may break through into the retro-pharyngeal space, and perforate the posterior wall of the pharynx as a retro-pharyngeal abscess.

Behind the tonsil is a peri-tonsillar space which is bounded posteriorly by the posterior pharyngeal fascia; between the posterior pharyngeal fascia and the prevertebral fascia is the retro-pharyngeal space, in which are located glands which are the seat of suppuration in retropharyngeal abscess. Lateral to the peri-tonsillar space and separated from it by pharyngeal fascia is the para-pharyngeal space; this space is bounded laterally by the parotid gland and within this space course the 9 th, 10th and 11th nerves, the ganglion of the superior cervical sympathetic, the internal carotid artery; adjacent to the parotid gland, the external carotid artery makes its course. In the lateral posterior corner of this space, the internal jugular vein may be seen. In these spaces are located the lymphatic glands which drain the Eustachian tube, posterior nose, middle ear and roof of pharynx. Many of these glands are not found in adults ; this explains the more frequent enlargement of deep jugular lateral lymph glands in children. The deep cervical glands course along the internal jugular vein and the internal carotid artery. These drain the mouth, tonsils, palate, pharynx, tongue, nasal fossæ, interior of skull, deep parts of head and neck, and when they suppurate they produce para-pharyngeal abscess.

In 1921 Kaufman pointed out that aspiration pneumonia may follow the spontaneous rupture of these abscesses. He thought that it was possible for mediastinitis, pleurisy and œdema of the larynx to develop as a result of these infections ; and clearly stated that small venous branches or the jugular its elfmay be the seat of thrombosis and give rise to pyæmia, the blood in such cases becoming infected and giving rise to metastases.

\section{Symptomatology.}

The symptomatology may be divided into several stages.

(1) Local symptoms due to primary infection (angina, Waldeyer ring inflammation, maxillary osteomyelitis, sinusitis, infection of teeth, infection of gums or floor of mouth, otitis, mastoiditis or venous sinus involvement, salivary gland involvement).

(2) Latent period.

(3) Symptoms due to involvement of neighbouring lymphatic glands, or to extension of the suppurative process within the immediate neighbourhood of portal or entrance of infection. (a) Simple hypertrophy of regional glands : abscess of regional glands; (b) hypertrophy of retro-pharyngeal glands : abscess of retro-pharyngeal glands ; (c) peri-tonsillar abscess ; (d) Ludwig's angina ; (e) para-pharyngeal abscess ; (f) phlegmon; (g) abscess extension by contiguity (gravitation abscess).

(4) Symptoms of sepsis : (a) with phlebitis of veins ; (b) with thrombosis of veins ; (c) without phlebitis and without thrombosis (?).

(5) Symptoms due to metastases.

(6) Symptoms due to retrograde thrombo-phlebitis: (a) by continuity ; (b) by embolism.

(7) Symptoms due to pressure.

(8) Symptoms due to erosion.

(9) Local symptoms. 
(1) Local symptoms.- The earliest symptoms are those of the primary infection. This may have its inception in any of the structures of the oronaso-pharynx. The severity of the primary infection has no definite relationship to subsequent development of sepsis ; cases severe and stormy at onset may make uneventful recovery without sepsis, and seemingly mild cases after apparent recovery may terminate fatally with septic manifestations. Apart from our general knowledge of the factors on which immunity and resistance to infection depend, the evolution of sepsis in these cases is not clear. The term post-anginal sepsis is a misnomer ; the infection may originate from lymphoid tissue (Waldeyer ring-lingual tonsil-palatine tonsils, pharyngeal tonsil, adenoids) ; from anywhere in the naso-pharynx ; from the bony maxillæ, teeth, and parasinuses ; from the gums and from all structures at the floor of the mouth; from the middle ear, Eustachian tube, mastoid and venous sinuses, and from the salivary glands.

(2) Latent period.-In those cases in which sepsis develops there is a latent period which varies from one to four days to from three to seven weeks following the initial infection. In this period many of the local manifestations of infection disappear, so that examination by inspection of local conditions is usually negative. Valuable information, however, may be gained by bimanual palpation, as deep abscesses and induration may only be discovered by this method of examination. Where there is a difference in the appearance in the parts affected on both sides, the part which looks the more pathological may not be the one responsible for the sepsis. The apparently normal looking tonsil or tonsillar area has been found on more than one occasion to be responsible for the sepsis, whereas the diseased looking area was not the primary cause of the sepsis.

(3) Extension symptoms.--The lymphoid tissue and lymph glands which drain the oro-naso-pharynx are the second line of defence against blood infections. It is natural to expect hypertrophy of the glands which drain the areas involved. Hypertrophy does not necessarily mean suppuration. In the majority of cases there is complete resolution without suppuration when the primary focus of infection is eradicated and properly met. In the minority of cases this hypertrophy goes on to suppuration. If the infection be mild and is properly met there is only hypertrophy followed by complete resolution. If the infection be of severe type or the patient of lowered resistance, the infection assumes the character of a phlegmon. This involves all tissues; induration and wema of local parts are marked; there are severe general symptoms, and unless the condition receives prompt and proper surgical attention the outcome is fatal in a very few days.

The seat of the primary infection determines the location of the second line of defence ; it is all a matter of lymphatic drainage. Depending on which locality is drained we have the following conditions to consider :-retropharyngeal abscess ; peri-tonsillar abscess ; Ludwig's angina ; para-pharyngeal abscess; abscesses which extend by gravity to structures below along lines of natural cleavage to the mediastinum. 
Retro-pharyngeal abscess.-This is a condition found almost exclusively in infancy and early childhood ; it is very rarely found in adult life ; about 7.5 per cent. of these patients are under one year of age. Its ætiological relationship to caries of vertebræ and foreign bodies is well understood, and will not be considered here. It may follow infection in the nose, middle ears, sinuses, teeth, mouth, pharynx and larynx.

The great susceptibility in infants is probably due to the presence of a larger number of glands in the post-pharyngeal space (3 to 10 or more); in adults there are rarely found more than one or two. The retro-pharyngeal glands receive afferent lymphatic vessels from para-sinuses, nasal fossæ, pharynx and larynx. These glands send efferent vessels to the internal jugular group of the superior deep cervical chain.

The vertebral, pharyngeal and para-vertebral glands are intimately associated with the adenoid vegetations. The symptoms are practically the same as those of peri-tonsillar abscess, except that the course is more prolonged and the pain is less. In children the onset is insidious and the symptoms are due to interference with deglutition, respiration and with speech (dysphagia, dyspnœea, rhinolalia). Respiratory interference is more common in children, because the larynx is located at least one vertebra higher than in adults and this in spite of the fact that the neck is proportionately longer. The cry is peculiar (cri de canard).

An irritable cough is usually present; and croup due to reflex spasm has been often mistaken for diphtheria. When the abscess assumes large proportions pressure symptoms develop, which if not relieved may prove immediately fatal. The diagnosis is often made on palpation when inspection is not satisfactory; if a definite fluctuating mass be felt, it should be immediately opened; otherwise there is danger of spontaneous rupture (due to manipulation) with fatal termination. A number of cases have been recorded, who succumbed suddenly after manipulation. Before opening an abscess it is most important to be sure that we are not dealing with an aneurysm. A colleague opened one with fatal outcome, when the aneurysm was mistaken for a retro-pharyngeal abscess. When opening these abscesses, a mouth gag should not be used. In the great majority of cases there is but little elevation of temperature ; in a small number it may be as high as $104-105^{\circ} \mathrm{F}$. It is in these cases that the cervical glands are particularly large, though they are enlarged to some extent in most cases ; the cocking of the head on the contrary is most apt to be seen when the retro-pharyngeal glands are especially enlarged.

Peri-tonsillar abscess.-This is rarely met in children, compared with the frequency of retro-pharyngeal abscess; but, it is not as rare as the literature would indicate. We have had three cases of peri-tonsillar abscesses in children under three years of age at one time. In the great majority of cases the abscess is antero-superior to the tonsil. In about 7 to 10 per cent. of cases it is bilateral. The symptoms are much the same as those of retropharyngeal abscess. Pain, fever, chill, dysphagia, dyspnœea, offensive breath, swollen lymphatic glands at angle of jaw, wedema of uvula (not seen in retro. 
pharyngeal abscess,) which is deviated to normal side, and cedema of the soft palate.

Para-pharyngeal abscess. - The para-pharyngeal space may become involved primarily, or may be the seat of suppuration secondary to peritonsillar abscess or retro-pharyngeal abscess. When suppuration occurs in this space, internal incision does not usually relieve the condition and external operation becomes necessary. Broca in 1903, pointed out that almost all acute para-pharyngeal abscesses are adeno-phlegmons and involve the chain of glands along the carotid sheath, as distinguished from those in front of the pre-vertebral muscles. These drain the mouth, tonsils, palate, pharynx, tongue, nasal fossæ, interior of the skull and deep parts of head and neck.

LUDWIG'S ANGINA.-Ludwig's angina is a phlegmonous process arising from infections within the floor of the mouth localized in a definite anatomic space. This space is secured by boundaries and has as its floor, the mylohyoid muscle ; as its lateral walls, the bodies of the mandible; as its posterior wall, the muscles which unite to form the base of the tongue and the deep part of the maxillary glands; and as its roof, the tongue and the muscles covering the floor of the mouth. The origin of the infections which develop into Ludwig's angina is within the lower gingival borders, usually around the teeth and the floor of the mouth. There is proof against the theory that the primary infection is in the submaxillary space. This area is drained by deep cervical glands alongside the carotid sheath.

GRAVITY ABSCESSES.-These abscesses in the throat may rupture spontaneously and drain into the pharynx ; they may burrow behind large vessels and the sterno-mastoid muscle and point in the posterior lateral triangle of the neck; they may rupture through the fascia and point anterior to the muscle in the anterior triangle; they may gravitate to lower parts of neck, under the clavicle and in the axilla (Pott's disease); they may travel downward behind the osophagus into the posterior mediastinum.

MaXillary SEPSIS IN INFANTS.-There have been reported about 32 cases of sepsis in infants following acute osteo-myelitis of the superior maxilla. In the majority of cases it has been mistaken for sinus disease. About 25 per cent. of cases die. It affects especially new born or very young infants. The infant suddenly becomes ill with fever and marked prostration; there are swelling and redness about the tissue of one eye. These are followed or accompanied by a purulent, unilateral nasal discharge, by swelling and softening of the alveolar border of superior maxilla on the affected side, and by pointing and the formation of abscesses on the hard palate, the alveolar border and often on the face between the eyes and nose. Sequestra and frequently small teeth are discharged from these sinuses. Such cases show high irregular fever; the blood culture is positive. The majority recover; others develop metastatic abscesses and succumb.

(4) Symptoms of sepsis.-These are so well known that we need not dwell long on this point. Fever is the outstanding symptom. It may be continuous or intermittent; and is usually associated with chills which are present 
in one-half the cases of sepsis. Chills at the onset of the initial infection are of no significance ; but when they reappear after the latent period, great importance must be attached to them, especially if they are repeated. The pulse is rapid, soft and variable; the skin is highly coloured or dusky to cyanotic; the spleen and the liver are enlarged ; diarrhoea is frequent ; vomiting is not often present. Drowsiness is present. The blood culture is positive in half the cases; a negative culture, is of no significance. The urine shows evidence of toxic irritation and a few red blood cells are usually found at every examination. The white blood cells are increased in number; the polymorphonuclear count is high. In adults, a state of euphoria has been noted many times.

In these cases of sepsis, there is primarily a phlebitis of a vein (mural thrombus). The pathological process may not go on beyond this and the patient may get well even in the presence of metastases, with surgical treatment to the local condition.

In a small number of cases, the phlebitis goes on to complete thrombosis, with the formation of an obturating thrombus. These cases must be recognized early and the condition must be properly met surgically if they are to be saved.

In another group of cases, the so-called fulminating type, there is no abscess formation, there is no phlebitis and there is no external cervical swelling. The body is overcome by a very rapidly advancing blood infection, and the condition proves fatal in three or four days.

(5) Metastases.--The local symptoms and signs may be so insignificant that the condition may be entirely overlooked, were it not for the appearance of metastases. Metastatic involvement of lung (multiple abscesses) is perhaps the most common metastatic lesion. The signs in the lungs are not clear cut and are often missed without X-ray examination of the chest. Empyema and pleurisy are frequent metastatic lesions. Metastatic lesions have been recorded in the endocardium, joints, kidneys, central nervous system, genital organs, eye, ear, bone-marrow, spleen and liver, skin, appendix, peritoneum, subphrenic abscess. These give symptoms peculiar to the organ involved.

The streptococcus causes metastases usually in the lungs, pleuræ, endocardium and joints, and these lesions suppurate in 25 per cent. of cases. The staphylococcus produces purulent metastases in 45 per cent. of the cases. The pneumococcus usually produces purulent meningitis and endocarditis.

(6) Retrograde thrombo-phlebitis.-Symptoms due to retrograde thrombophlebitis are usually due to thrombosis of the internal jugular vein and the venous sinuses which directly or indirectly open into it. Thus we may have thrombosis of lateral, cavernous and petrosal sinuses; in fact, we have once seen all the venous sinuses of the head become thrombosed. Basilar and general meningitis have been recorded many times; this may take place by direct extension from the para-pharyngeal space or by continuity or by embolism through the venous sinuses. Abscess of the brain and encephalitis have also been noted as complications of this condition. 
Wessely in experimental injections of tonsils with Indian ink, proved that there is a direct channel from the tonsils into the para-pharynx, and from there to the base of the brain to foramen ovale. He thus explains the spread of infection to the brain and meninges in these cases by direct extension. He believes that the infection may spread to the brain through the loose tissue in the para-pharyngeal space, through venous anastomoses, or by both routes.

The first case with cerebral complications was reported by Stanislaus v. Stein (Moscow) in 1885. He reported a case of acute phlegmonous pharyngitis, complicated by purulent meningitis. Other cases with meningeal complications have been recorded by Tollen (1903), cavernous sinus thrombosis-purulent basilar meningitis ; Jacques and Lucien (1908) cavernous sinus thrombosis; Kandler (1907) abscess of brain; Proskauer (1914) purulent hæmorrhagic inflammation of dura and circumscribed lepto-meningitis; Fall (1919) abscess followed by osteo-myelitis, necrosing osteo-myelitis and meningitis; Höstorn (1920) cavernous sinus thrombosis and basilar meningitis; Beck (1914) suppurative meningitis, extra-dural abscess.

(7) Pressure symptoms. - The symptoms which may be expected as a result of pressure in this locality become clear if we refer to the anatomy of the parts. In the neighbourhood of the para-pharyngeal space are the internal jugular vein, the 9 th, 10 th and 11th nerves, the ganglion of superior cervical sympathetic, the hypoglossal nerve; and the alveolar and facial nerves are in close proximity to the parotid, which not infrequently becomes involved in the abscess formation. Hence symptoms of vagus irritation, and irritation of the 9th and 1lth nerves may be found. Pressure may be exerted on larynx, œesophagus, thyroid, trachea, lung; therefore difficulty in swallowing, voice changes, hoarseness, dyspnœa (air-hunger) may result. A symptom often present in these cases is opisthotonos which leads to a mistaken diagnosis of meningitis. This is probably due to irritation of spinal nerves by enlargement of, or abscess in, the retro-pharyngeal glands.

(8) Erosion symptoms.-Symptoms may arise suddenly from erosion of vessels or from rupture of an abscess into various organs, as ear, pharynx, larynx, lung, mediastinum, or intra-cranially through the internal auditory canal. When erosion of blood vessels occurs, it usually involves the internal carotid artery or branches of the external carotid artery (lingual, facial, or ascending pharyngeal).

(9) Local symptoms.- These may play only a secondary role in the establishment of a correct diagnosis. In the throat there may be but slight evidence of present or past disease. One side of the throat may appear more nearly normal than the other, and yet may be the cause of jugular thrombosis. In the cases in which retro-pharyngeal or peri-tonsillar abscesses or Ludwig's angina are present, the local appearances are quite characteristic. In the great majority of cases, there is external swelling of the neck (lymphatic glandular enlargement) or brawny induration as in Ludwig's angina ; deep pain at angle of jaw is usually present and may be elicited on pressure; in the neck an indurated strand following the anterior border of the sterno-mastoid muscle may be felt. In many cases trismus is marked. The odour of the mouth is foul. An exudate on the tonsils and fauces may be present which makes it imperative to differentiate this condition from acute leukæmia, agranulocytic angina, monocytic angina, Vincent's angina and diphtheria. 


\section{X-ray examination.}

An X-ray examination should always be made to rule out foreign body, caries of vertebræ and mastoiditis. In not a few cases an abscess which was not suspected or which could not be seen or felt, was discovered by the use of the X-ray.

\section{Bacteriology.}

In many cases the anærobic streptococcus putrificus was isolated from the local abscess and from the blood. Many other organisms have been responsible for the condition; streptococcus hæmolyticus, streptococcus viridans with influenza bacillus, staphylococcus albus, etc. S. putrificus especially causes a foul local odour, a rapid course, and metastases, preferably in the lung. The ærobic bacteria metastatize preferably in the joints, muscles, skin, and not so frequently in the lung; the course is not so stormy though it may last for weeks ; these cases usually get well when internal jugular is ligated.

The anærobic bacteria are more apt to invade venous channels, whereas the ærobic first invade the lymphatics and then the blood vessels.

\section{Frequency and prognosis.}

Post-anginal sepsis is far more frequent than the case reports and the literature suggest. We have had four cases of the condition in children at the hospital at one time. It is far more frequent in children than the literature indicates. We have had 10 cases (with three deaths) in children under 10 years of age in less than 18 months.

One hundred and thirty-seven cases of post-anginal sepsis were collected by Uffenorde in 1928. Of these 87 were males and 50 females, and 3 were under 10 years of age, the youngest being 5 years. In mild cases the condition may last only 3-8 days after the latent period. In fulminating cases, without visible pathology, and in phlegmonous cases with much induration and œdema, the outcome may be fatal in 3-4 days. In the majority of cases the course lasts from 8-21 days or longer, depending on the number and severity of metastases.

Most authors have found the lesions more frequently on the left than on the right side. Waldapfel in 43 cases noted that the lesions were on the right side in 13 cases, on the left in 20 cases, and bilateral in 10 cases.

Mortality.-In 137 cases collected by Uffenorde, only 40 recovered or were cured by operation. Reye reported 12 deaths in 17 cases; Waldapfel 25 deaths in 43 cases; Claus 14 deaths in 28 cases. In Waldapfel's series, of the 25 fatal cases, 17 were operated on and 8 were not; of the 18 surviving cases, 15 were operated on, and 3 were not.

\section{Diagnosis.}

The diagnosis of post-oro-naso-pharyngeal sepsis rests on the following points :-

(1). The history is of utmost importance. In almost every case there is a previous history of angina, tonsillitis, tooth infection, sinus disease, otitis, parotitis, gingivitis, adenoiditis, etc. 
(2). In the majority of cases there is a latent period following the primary infection, varying from one day to one month.

(3). The sudden onset of septic symptoms with recurrent chills and in many cases positive blood culture.

(4). In the great majority of cases external swelling of the neck is present.

(5). The presence of metastases in lung, pleura, joints, heart, meninges, venous sinuses, etc.

(6). The local lesion may appear fairly normal or show but slight disease. In cases where the lesions are not apparent on inspection, information can be obtained by bimanual palpation and by X-ray examination. In the majority of cases there is evidence of peri-tonsillar, retro-pharyngeal or parapharyngeal abscess.

(7). Exploratory operation and inspection of the internal jugular vein is indicated in every case presenting the above symptoms which has a positive blood culture and presents a cord-like thickening along the sterno-cleidomastoid muscle.

Differential Diagnosis.-In every case of foul smelling angina we must think of acute leukæmia, agranulocytic angina, monocytic angina, Vincent-Plaut's angina, and diphtheria.

In those cases in which there is a grey foul-smelling exudate on tonsils and pharyngeal walls it is safer to administer antitoxin before the report of the culture is known.

In leukæmia, especially in acute lymphatic type, there is often a necrozing infiltration of the tonsils with foul odour. The blood when characteristic usually renders the diagnosis simple ; but in many cases, the blood picture may be aleukæmic and the differential count not characteristic. In these cases there may be general adenopathy, symptomatic purpura, negative blood cultures, much greater splenic and hepatic enlargement ; the course is longer ; and biopsy of an affected gland will establish a definite diagnosis.

Agranulocytic angina is rare in children; although instances in children have been recorded by Chrisloff, Weiss, and Dwyer and Helwig. In this condition there is a necrozing inflammation of gums, tonsils and mucous membrane of the mouth and other mucous membranes (stomach, intestines, vulva). In association with this there is a leukopenia with a marked diminution to total disappearance of polymorphonuclears. There is little if any enlargement of the regional lymph nodes, and in about half the cases, icterus is present. This condition is to be differentiated from a clinical and blood picture which terminates leukæmia and sepsis. As favourable results have been reported by Friedemann with X-ray tests, cases should be given this therapeutic test.

Infectious mononucleosis, described in 1920 by Sprint and Evans is a self-limited disease in which there is a natural tendency to recovery within 2 to 3 weeks ; it is characterized by a transient lymphocytosis, fever, enlarge. ment of the spleen and lymph glands. The tonsils may be covered with a diphtheria-like membrane; they have no general symptoms of sepsis. 
In Vincent's angina, the ulcerations and the finding of the organisms (spirilla and bacilli) in pure culture is diagnostic. In all throat conditions Vincent's spirilla may be found in conjunction with other organisms. In Vincent's angina, the polymorphonuclears may decrease to 50-60 per cent. These cases improve rapidly on arsenical treatment internally and locally.

\section{Surgical treatment.}

It is our belief that in the majority of cases there is no obturating thrombosis of the large veins, but only phlebitis or mural thrombosis; we may therefore expect recovery in the majority of cases when the primary focus of infection is eradicated, and when surgical metastases are properly met, without any surgical treatment of the internal jugular veins or other venous sinuses. When the first signs of thrombosis of venous sinuses appear, the condition must be met promptly surgically. We believe that the German methods are too radical, as is perhaps reflected in the high mortality which is reported from that country. We are in perfect accord with them, however, in the belief that early incision of external swellings even when there is no fluctuation to be found is necessary. The results in such cases are excellent, even though no pus may be found at time of operation. The greater and the earlier the external swelling appears, and the earlier it is met surgically (before it breaks through into a vein), the more hopeful is the prognosis. Hippocrates recognized this many years ago ; it is true to-day.

Weiss and Haenschell have reported prompted recoveries in post-anginal sepsis after removal of tonsils in acute stage ; this undoubtedly proves that in a small number of cases the portal of entry and focus of infection are identical. Uffenorde attacks the focus of infection from the outside; he lays open the jugular sheath and exposes the para-pharyngeal and retro-pharyngeal spaces; the internal jugular is ligated; then enucleation of tonsils is carried out. Zange, attacks the tonsils first and ties off the internal jugular; then he cleans out all lymphatic glands in neck. ('laus, ligates the jugular; traces the facial veins to the tonsils and ties them off ; then enucleates tonsils. Clairmont has suggested an operation which in Germany is called 'Kollare Mediastinomie' ; this consists of cutting off the lymphatic and venous channels which drain into the mediastinum. In cedema of larynx secondary to cervical abscess in these cases, Leider, without tracheotomy, obtained good results by opening lymphatic channels in neck (on the jugular) after extirpating the swollen lymphatic gland.

We have records of eight cases of sepsis following oro-naso-pharyngeal infection ; three of these patients died ; four completely recovered ; one left the hospital still running a temperature. In one case a definite sinus thrombosis (involving all venous sinuses) was demonstrated at autopsy, and the following metastatic lesions were noted, pneumonia, mastoiditis, cervical abscess, parotitis, endocarditis, peritonitis. In the two other cases which died no autopsies were performed.

The German belief that in all these cases there is a thrombosis of the internal jugular or of its large anastomosing branches is not borne out by our experience; we believe that in all these cases there is thrombosis of small venules which acts as a focus of sepsis, but that these thromboses do not necessarily extend to the larger veins. In the majority of cases the thrombosis does not extend beyond the small veins. We feel that the suggestion that in post-anginal sepsis the internal jugular should always be tied is too radical 
a measure. We believe that the local condition and infection should be eradicated first ; if the symptoms persist, then it would seem that ligation of internal jugular should be carried out. In a number of cases the mere enucleation of the tonsils in the course of sepsis, caused a subsidence of temperature and complete recovery.

It is of primary importance to incise and lay free all exudative swellings in the neck, and clean out all.infected glands, in the presence of a post-anginal sepsis, even if there be no definite fluctuation to be ascertained.

The cases in which a cord-like swelling is felt in the neck along the margin of the sterno-cleido-mastoid muscle, or in any case which presents evidence of venous sinus or meningeal involvement, the ligation of the internal jugular should be carried out at once. 